Revue québécoise de psychologie

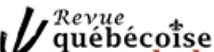

de psychologie

\title{
DEUX MODÈLES D'INTERVENTION EN TROUBLE GRAVE DU COMPORTEMENT (TGC) : COMPARAISONS ET AMÉLIORATIONS POSSIBLES
}

\section{TWO MODELS OF INTERVENTION IN SERIOUS BEHAVIORAL DISORDER (SBD): COMPARISONS AND POSSIBLE ENHANCEMENTS}

\author{
Guy Sabourin et André Lapointe
}

Volume 39, numéro 2, 2018

LES MEILLEURES PRATIQUES CLINIQUES PRÉCONISÉES DANS LE SECTEUR DE LA SANTÉ ET DES SERVICES SOCIAUX EN MATIÈRE DE PROBLÉMATIQUES COMPORTEMENTALES

THE RECOMMENDED BEST CLINICAL PRACTICES IN THE HEALTH AND SOCIAL SERVICES SECTOR REGARDING BEHAVIOURAL ISSUES

URI : https://id.erudit.org/iderudit/1051226ar

DOI : https://doi.org/10.7202/1051226ar

Aller au sommaire du numéro

Éditeur(s)

Revue québécoise de psychologie

ISSN

2560-6530 (numérique)

Découvrir la revue

Citer cet article

Sabourin, G. \& Lapointe, A. (2018). DEUX MODÈLES D'INTERVENTION EN TROUBLE GRAVE DU COMPORTEMENT (TGC) : COMPARAISONS ET AMÉLIORATIONS POSSIBLES. Revue québécoise de psychologie, 39(2), 161-190. https://doi.org/10.7202/1051226ar

\section{Résumé de l'article}

Le présent article vise à comparer deux modèles d'intervention en TGC, soit le modèle québécois d'Analyse et d'Intervention MultiModales (AIMM) et le modèle de Soutien Comportemental Positif (SCP : Positive Behavioral Support) chez les personnes présentant une déficience intellectuelle (DI). Ce modèle de LaVigna et Willis (2012) a d'ailleurs fait l'objet récemment de démonstrations convaincantes sur le plan de son efficacité et de son efficience clinique. Après avoir constaté les compatibilités et les différences entre ces deux modèles sur le plan qualitatif, certaines pistes d'amélioration clinique et de recherche concernant l'AIMM sont relevées. 


\section{DEUX MODÈLES D'INTERVENTION EN TROUBLE GRAVE DU COMPORTEMENT (TGC) : COMPARAISONS ET AMÉLIORATIONS POSSIBLES}

TWO MODELS OF INTERVENTION IN SERIOUS BEHAVIORAL DISORDER (SBD): COMPARISONS AND POSSIBLE ENHANCEMENTS

Guy Sabourin ${ }^{1}$ SQETGC

Dans le domaine de la déficience intellectuelle (DI), l'intérêt au Québec pour le concept de trouble grave du comportement (TGC) a augmenté de façon importante depuis les vingt dernières années avec la mise sur pied de programmes spécifiques pour cette problématique. II existe, à notre connaissance, deux modèles d'intervention importants dans le domaine, soit l'Analyse et l'Intervention MultiModales (AIMM) et le Soutien Comportemental Positif (SCP). Le présent article vise à présenter brièvement ces modèles ainsi que les études d'efficacité associées. Après avoir relevé un certain nombre de compatibilités et de différences, des pistes d'amélioration et de recherche sont discutées.

Dans la région de Montréal, il y a eu, en 2000, la création d'un programme intitulé "Programme régional d'expertise multidisciplinaire en troubles graves du comportement (PREM-TGC) $»^{2}$. À la suite de divers processus de consultations professionnelles auprès d'experts dans le domaine, ce programme a adopté le modèle d'intervention inspiré des travaux de Griffiths, Gardner et Nugent (1998) et il porte, au Québec, l'appellation d'Analyse et d'Interventions MultiModales (AIMM).

Il est important de rappeler qu'il n'existe pas de consensus clair sur la définition de trouble du comportement chez les experts. Des travaux réalisés au Québec ont toutefois permis de dégager un consensus sur la notion de trouble grave du comportement auprès d'une soixantaine de professionnels dans le domaine (Tassé, Sabourin, Garcin et Lecavalier, 2010). Selon ce consensus, les comportements problématiques (CP) d'une personne sont jugés graves par l'environnement social lorsque son trouble du comportement (agression physique, automutilation, comportement destructeur, etc.) met en danger, réellement ou potentiellement, l'intégrité

1. Adresse de correspondance: 964 , rue des Sitelles, Mont-St-Hilaire (QC), J3H 5T5. Téléphone : 514-922-3720. Courriel : bureau@guysabourinphd.com

2. Le concept de trouble grave du comportement n'est pas considéré actuellement au Québec comme un diagnostic formel, mais plutôt comme un descripteur clinicoadministratif permettant de mieux décrire la situation clinique d'une personne et de lui offrir dans le réseau public des services spécialisés ou surspécialisés pour répondre plus adéquatement à ses besoins particuliers (SQETGC, 2017). Par souci de cohérence, le concept descriptif de trouble grave sera utilisé au singulier dans le texte, sans postuler un caractère nosologique de trouble de santé mentale. 
physique ou psychologique de la personne, d'autrui ou de l'environnement, compromet sa liberté, son intégration ou ses liens sociaux (Tassé et al., 2010). À partir de cette définition, une évaluation de la prévalence de TGC à un moment dans l'année 2014 a pu être établie à $6,8 \%$ pour l'ensemble de la clientèle des centres de réadaptation en déficience intellectuelle et en troubles envahissants du développement (Guimond, Paquet et Bigras, 2016). Ce taux est plutôt faible, mais les conséquences et impacts de leur présence peuvent être désastreux pour la personne et son entourage (Allen et al., 2013; Tassé et al., 2010).

Griffiths et ses collègues (1998) désiraient proposer un modèle d'intervention fonctionnelle et personnalisée de différentes méthodes d'intervention comportementale à utiliser auprès des personnes présentant une DI qui manifestaient simultanément un trouble du comportement (TC) et des problèmes de santé mentale. Gardner (2002) a par la suite appliqué ce modèle aux comportements agressifs chez ces personnes. Cette problématique comportementale est d'ailleurs très fréquente chez cette clientèle et est souvent considérée comme un TGC étant donné la gravité, la chronicité et les impacts de ce type de comportement (Gardner, Došen, Griffiths, King et Lapointe, 2006).

Dans le cadre des travaux montréalais sur les TGC amorcés vers les années 1995, les $D^{\text {rs }}$ Griffiths et Gardner ont été appelés, à titre de consultants et d'experts en TC, à intervenir auprès de plusieurs personnes manifestant un TGC. Ils ont par ailleurs également donné de la formation et offert de la supervision et du coaching à divers professionnels. C'est dans ce contexte que leur approche multimodale fonctionnelle contextuelle a été adoptée par l'équipe de professionnels associée au Programme régional d'expertise multidisciplinaire en TGC de Montréal (PREM-TGC) comme modèle clinique de base en analyse et en intervention en TGC. Par la suite, ce modèle clinique s'est répandu à travers le Québec et a même été intégré dans le Guide de pratique de la Fédération québécoise des centres de réadaptation en déficience intellectuelle et en troubles envahissants du développement en 2008 (FQCRDITED, 2010) pour améliorer les interventions en TGC de cette clientèle.

Postulats et principes de base du modèle

L'analyse et l'intervention multimodales (AIMM) telle qu'adoptée à la FQCRDITED réfère, selon Sabourin et Lapointe (2014), à un cadre clinique et théorique intégrateur inspiré des meilleures pratiques permettant d'expliquer et d'intervenir en TGC chez les personnes présentant une DI avec ou sans trouble du spectre de l'autisme. Ce cadre vise à soutenir la prise de décision, par une analyse des causes les plus probables d'un TGC, sur les meilleurs services d'adaptation et de traitement, mais aussi sur la mise en place de soutien requis, de façon 
individualisée, dans une perspective de prévention des TGC. L'AIMM repose sur des principes de base qui guident le choix de cibles à atteindre, les méthodologies retenues, les décisions à prendre (hypothèses de travail) et les interventions qui en découlent. Ces principes sont au nombre de huit (8), couvrent l'ensemble des postulats et des valeurs à respecter dans le processus d'AIMM.

\section{Principe $n^{\circ} 1$ : Présence de causes multiples fonctionnelles et contextuelles en TGC}

Les TGC ont généralement des causes multiples. Ces causes doivent être comprises comme étant surtout contextuelles (fortement influencées par l'environnement), mais aussi par des causes plus distales d'ordre biopsychosociales, influencées soit par des troubles génétiques, de santé physique ou de santé mentale, par l'histoire personnelle et les traits psychologiques de la personne ou des difficultés neuropsychologiques ou même neurologiques (Gardner et al., 2006; Sabourin et Lapointe, 2014). Selon Došen et ses collaborateurs, un TGC serait le résultat de l'interaction défavorable des caractéristiques de la personne avec celles de son environnement et qui apparaît dans un ou des contextes particuliers (Došen, Gardner, Griffiths, King et Lapointe, 2007).

\section{Principe $n^{\circ} 2$ : Nécessité d'une évaluation fonctionnelle globale des causes}

Dans le but de pouvoir intervenir efficacement, il est nécessaire d'évaluer l'ensemble des causes (sources d'influence externe et interne sur l'apparition et le maintien) de TGC relativement à des stimuli contextuels et à des moments spécifiques. Cette méthodologie permet de développer des hypothèses explicatives plus exhaustives du TGC. Elle favorise aussi la sélection d'interventions les plus efficaces afin de réduire l'influence des causes sous-jacentes de ces comportements (Gardner et al., 2006; Gardner, Griffiths et Hamelin, 2012). Pour ce faire, une équipe transdisciplinaire est généralement requise pour faciliter le dépistage des causes multiples et complexes qui peuvent contribuer au maintien d'un TGC (Guide de pratique en TGC de la FQCRDITED, 2010).

\section{Principe $n^{\circ} 3$ : Interventions simultanées sur les diverses causes}

Sur le plan de l'intervention comme telle, il est nécessaire d'agir de façon simultanée à divers niveaux pour atteindre une efficacité optimale étant donnée la présence de causes multiples du TGC. II s'agit d'adopter une perspective préventive en agissant, en même temps, sur les diverses causes et non en intervenant uniquement sur le comportement problématique lorsqu'il apparaît ou qu'il est sur le point d'apparaître. Ainsi, il serait pertinent, entre autres, de diminuer la présence des facteurs contextuels dans l'environnement de la personne, mais aussi d'augmenter ses compétences, car la personne peut présenter de nombreuses 
difficultés sur le plan adaptatif qui contribuent à la probabilité du TGC. On devrait prioriser l'apprentissage d'habiletés qui manquent à la personne dans les contextes d'apparition les plus à risque.

\section{Principe $n^{\circ} 4$ : Planification centrée sur la personne et sa qualité de vie}

De façon plus globale, les décisions cliniques concernant la personne doivent être prises sur la base de ses intérêts, de ses motivations et de l'amélioration de sa qualité de vie. La personne et son entourage participent à toutes les décisions la concernant. En misant le plus possible sur les capacités d'autodétermination (choix, options, etc.) de la personne, il sera envisageable de faciliter sa collaboration dans chacune des étapes d'intervention, de l'évaluation à l'adaptation, à la réadaptation ainsi qu'au traitement.

\section{Principe $n^{\circ} 5$ : Décisions cliniques fondées sur l'approche positive (Fraser} et Labbé, 1993)

Les moyens d'évaluation et d'intervention retenus doivent être respectueux et significatifs pour la personne afin de donner une valeur au projet de vie qui le sous-tend (le plus possible communautaire et valorisant). II en est de même pour son entourage qui l'accompagne dans son cheminement pour l'atteinte de ses buts. En fait, la planification des interventions doit être globale, c'est-à-dire capable d'intégrer, le plus possible, tous les principes directeurs, tout en étant systémique dans l'intention de tenir compte de la qualité de vie de la personne, mais aussi de celle de l'environnement dans lequel la personne évolue. Pour ce faire, la concertation et la participation de la personne et de son entourage aux décisions sur les causes et les moyens d'intervention doivent être optimales (Gimpel Peacock et Collett, 2010).

Principe $n^{\circ} 6$ : Nécessité d'un plan écrit et systématique comportant des services intensifs de prévention de crise, de traitement et de soutien

Pour ce faire, le processus d'intervention doit être systématique et documenté sur le plan de l'évaluation, de la planification, de l'intervention et de son ajustement jusqu'à l'évaluation des résultats plus globaux de la démarche d'AIMM (Gardner et al., 2006). La rigueur et une démarche réflexive dans le travail d'analyse et d'intervention sont donc nécessaires pour objectiver la prise de décision dans un document écrit (p. ex., Plan d'action multimodal en TGC - PAMTGC). Pour ne pas se fier à des jugements subjectifs ou à des perceptions pour prendre des décisions cliniques, il vaut mieux appuyer les décisions concernant la personne et son entourage sur des données plus objectives d'observation, le plus possible de façon continue. Ce processus rendra plus faciles l'adhésion et la collaboration de l'entourage ainsi que de l'ensemble des partenaires aux hypothèses de travail retenues et aux moyens d'intervention sélectionnés. 
Compte tenu de l'ampleur et de la complexité des besoins des personnes présentant un TGC, la réponse à ces besoins nécessite une haute intensité de services personnalisés et composés d'interventions de gestion préventive, de modalités de gestion de crise, d'adaptation ou de traitement ainsi que de divers soutiens appropriés. Tous ces services et soutiens doivent être intégrés dans un plan d'intervention (PI) global et bien coordonné pour en favoriser l'efficacité optimale.

\section{Principe $n^{\circ} 7$ : Les interventions en milieu naturel ou communautaire sont à privilégier}

Ces services et soutiens doivent être offerts, le plus possible, dans le milieu naturel de la personne, de la façon la moins intrusive et la moins contraignante. Ceci permet de sauvegarder une meilleure qualité de vie et de pouvoir rapprocher l'expertise (et les soutiens) de la personne et de son entourage. De plus, l'utilisation de ce principe tend à éviter l'institutionnalisation des services et à ne pas éloigner la personne de contextes significatifs d'apprentissage et de développement de sa qualité de vie.

\section{Principe $n^{\circ} 8$ : Nécessité d'un système proactif de services}

Des conditions organisationnelles essentielles doivent être mises en place pour pouvoir utiliser et actualiser ces principes dans des services publics de santé et de services sociaux. Pour atteindre un niveau de qualité intéressant de services aux personnes manifestant un TGC, il faut un engagement organisationnel significatif de la direction des établissements concernés, en particulier de celle responsable de la coordination des services (Gardner et al., 2006; Roberge, 2015). Cela est nécessaire pour garantir une bonne sélection, une préparation, une formation optimale et un soutien continu à l'ensemble des ressources pertinentes à la réponse aux besoins de cette clientèle. Un tel engagement organisationnel devrait aussi favoriser une meilleure ouverture du personnel au niveau des valeurs sous-jacentes au modèle d'intervention. De plus, l'engagement des directions d'établissements devrait inclure de réaliser l'évaluation des résultats de ces services non seulement en termes de réduction d'un $T G C$, mais également d'augmentation de leur qualité de vie et de la réduction des situations de handicaps (Guide de pratique en TGC de la FQCRDITED, 2010).

\section{Processus d'analyse et d'intervention}

Rappelons que l'AIMM est un cadre clinique et théorique intégrateur qui s'inspire des meilleures pratiques permettant d'expliquer la présence de TGC chez les personnes présentant une DI ou un TSA. Ce cadre vise à soutenir la prise de décision des services d'adaptation et de traitement requis, mais aussi sur le plan des services de soutien requis de façon 
individualisée, et ceci, par une analyse systématique des causes fonctionnelles probables dans une perspective de prévention. Ce cadre a été développé au Québec sous la forme d'un processus clinique reproductible et applicable à toutes les personnes présentant une DI avec ou sans TSA et en situation de TGC. Celui-ci est composé essentiellement de sept grandes étapes séquentielles et relativement uniformisées (associées à des méthodologies particulières) favorisant une utilisation systématique et conforme aux principes sous-jacents du modèle préconisé.

Ces étapes sont les suivantes :

1. Décrire le ou les troubles graves du comportement;

2. Recueillir et organiser l'information pertinente sur les causes (les plus probables);

3. Formuler des hypothèses globales et causales;

4. Élaborer des interventions sur mesure;

5. Organiser le suivi de ces interventions (en planifiant les moyens et en définissant les responsabilités);

6. Intervenir;

7. Faire le suivi régulier des interventions priorisées (pour ajuster, le cas échéant, les hypothèses et les interventions qui en découlent).

Un protocole récent élaboré par le SQETGC (SQETGC, 2017) précise les diverses modalités d'évaluation recommandées pour attribuer à un usager le descripteur clinico-administratif de TGC pour les personnes présentant une DI avec ou sans TSA. Dans la première étape du processus, il s'agit, entre autres, d'identifier quels sont les types de CP (mesurables et observables) qui sont présents chez la personne dans les derniers mois, leur niveau de fréquence et de gravité en termes de conséquences et d'impacts, et ceci, avec un instrument de mesure standardisé (p. ex., Échelle d'évaluation globale de la gravité des comportements problématiques - version deux révisée - EGCP-II-R: Sabourin, Senécal et Paquet, 2016) ou selon l'avis d'une équipe professionnelle. Ceci devrait permettre d'identifier la présence de TGC et le profil de TC, mais surtout quel TGC devrait faire l'objet, en priorité, d'une analyse multimodale (approfondie). Comme il s'agit d'identifier les causes d'un TGC, le fait d'agir sur les causes du TGC le plus important permet de diminuer de façon significative l'ensemble des TC de la personne, car, souvent, plusieurs TC ont les mêmes causes sous-jacentes (Sabourin et Lardon, 2006).

En second lieu, il faut recueillir et organiser l'information pertinente. Pour recueillir toutes ces informations, il faut savoir quoi rechercher et comment la synthétiser. Dans le modèle de l'AIMM, les éléments de base dans la ligne temporelle des causes du TGC sont à examiner et à 
documenter. Ces éléments doivent faire l'objet d'observation systématique de façon naturelle ( $p$. ex., fiche $A B C$ ), de façon rétrospective (questionnaire, entrevues, lecture du dossier) ou par une analyse expérimentale. Cette ligne temporelle est représentée comme suit: Facteurs de vulnérabilité $\rightarrow$ Facteurs contextuels $\rightarrow$ Traitement de l'information $\rightarrow$ TGC $\rightarrow$ Facteurs de renforcement

Avec les diverses observations et cueillettes de données, on cherche surtout à réaliser une analyse fonctionnelle, c'est-à-dire documenter les liens probabilistes entre les facteurs contextuels (antécédents: déclencheurs et contributeurs), le TGC (comportement cible) et les facteurs de renforcement (conséquents). II y a d'ailleurs des méthodologies bien connues et reconnues pour ce faire (voir Coffre à outils de l'éducateur en TC/TGC, 2015).

Une fois la cueillette de données amorcée, les informations obtenues sont insérées sous forme de résumé dans la grille d'analyse multimodale contextuelle. Cette grille permet de synthétiser l'ensemble de ces informations et de faciliter la formulation d'hypothèses exhaustives des causes du TGC ciblé. Elle favorise la visualisation des nombreuses interactions entre les éléments de causalité possibles par les membres de l'équipe de la personne (incluant la personne et son entourage). Sur le plan horizontal, on retrouve les différents facteurs de la ligne temporelle de causalités décrites plus haut. En vertical, un ensemble de huit dimensions sont identifiées, dont trois pour décrire l'environnement de la personne (appelé contexte externe d'influence) et cinq différents éléments psychologiques et physiologiques pertinents (appelé contexte interne d'influence chez la personne).

Pour faciliter ce travail de cueillette de données et de sélection d'informations pertinentes par les intervenants ou les professionnels concernés, des inventaires de causes probables d'un TGC ont été élaborés pour des clientèles spécifiques, DI ou DI avec TSA (Morin, Sabourin, Labbé et Beaulieu, 2015). Compte tenu des contraintes liées à la publication dans la présente revue, nous ne pouvons décrire de façon détaillée les concepts sous-jacents de la grille multimodale ${ }^{3}$. II est important toutefois de présenter les dimensions traitées dans la grille. II s'agit, dans le contexte externe, de l'influence potentielle sur la personne de son environnement physique, de son environnement social et, enfin, de ses habitudes et de son style de vie. Le contexte interne d'influence

3. Les lecteurs intéressés pourront se référer aux travaux approfondis en 2014, réalisés en collaboration avec la communauté de pratique provinciale en TGC, lesquels ont permis de définir les 13 concepts de base de la grille de façon à ne retenir que les éléments les plus aidants pour dégager une lecture fonctionnelle des causalités du TGC (Sabourin et Lapointe, 2014). 
regroupe des éléments sur les caractéristiques psychologiques de la personne (non pathologiques), ses habiletés déficitaires, sa santé physique, sa santé mentale et, finalement, sur ses caractéristiques neurologiques ou neuropsychologiques.

Dans le processus d'analyse multimodale, seules les informations en lien direct avec le TGC seront retenues dans la grille. Par exemple, la présence d'irritabilité (santé mentale) et de fatigue (santé physique) subséquentes à une crise d'épilepsie (aspect neurologique) seront inscrites dans la grille relativement à un TGC de frapper l'intervenante (le TGC) qui a fait une demande insistante (facteur contextuel). Le diagnostic de $\mathrm{DI}$ légère de cette personne pourrait ne pas être mentionné dans la grille s'il n'a aucun lien apparent avec le TGC analysé. La grille multimodale vise donc à être un outil synthétique évolutif en fonction des observations et des évaluations réalisées par l'entourage et par les professionnels concernés. La grille s'avère aussi très utile pour déduire de l'analyse des interactions entre les différents éléments relevés d'autres causalités plus complexes ou plus difficiles à observer directement.

La troisième étape consiste à élaborer des hypothèses causales globales à partir des informations recueillies dans la grille multimodale. Selon Gardner (2002), l'identification de différentes hypothèses globales permet une formulation de cas plus significative (pour une compréhension holistique des TGC de la personne). De plus, elles favoriseraient une plus forte adhésion aux recommandations d'intervention qui en découlent par l'entourage de la personne. Certaines hypothèses causales devront être priorisées pour choisir les interventions à implanter. Les hypothèses seront sélectionnées en fonction des éléments jugés comme les plus susceptibles de contribuer, en fréquence ou en importance, à l'apparition du TGC. Par exemple, des hypothèses spécifiques prendront la forme de : "Lorsque (tels facteurs contextuels), parce que (tel facteur de vulnérabilité ou telle réaction du traitement de l'information de la personne est présent), le comportement $X$ se manifeste, pour obtenir ou éviter (tel facteur de renforcement) ॥ (Formation AIMM-SQETGC, 2014). Ce type d'hypothèses favorise la sélection d'objectifs et de moyens en lien direct avec des causes fonctionnelles de TGC, ce qui en augmente la probabilité d'efficacité selon divers travaux en ce sens (Harvey, Boer, Meyer et Evans, 2009).

La quatrième étape consiste à élaborer des interventions sur mesure. Les trois composantes des interventions sur mesure sont les aménagements préventifs, la prévention active et les interventions de réadaptation et de traitement. L'AIMM postule que pour obtenir des résultats optimaux en TGC, il faut intervenir à ces trois différents niveaux. 
La première composante vise à réduire le plus rapidement possible la probabilité d'apparition de TGC dans une perspective de prévention afin de rendre la personne accessible à de nouveaux apprentissages et de réduire les impacts négatifs générés par le TGC pour la personne elle-même, mais aussi pour son entourage. Ces changements ont pour cible la modification des contributeurs, des déclencheurs et des facteurs de renforcement. II s'agit donc de moduler les facteurs externes de l'environnement de la personne, d'où son appellation d'aménagements préventifs. Pour ce faire, quatre grandes stratégies sont recommandées, soit :

- Retirer ou réduire l'influence des facteurs contextuels (les contributeurs et les déclencheurs);

- Introduire des conditions favorisant des comportements alternatifs;

- Modifier les facteurs de renforcement;

- Modifier le style de vie de façon globale (Formation AIMM-SQETGC, 2014).

Les aménagements préventifs n'impliquent pas de nouveaux apprentissages de la personne, mais bien la modulation des conditions environnementales pour réduire la probabilité des TGC. On pense ici à changer les façons habituelles d'agir avec la personne (demandes, instructions), à ajuster les exigences, à introduire de nouvelles sources de stimulation, à améliorer son style de vie (offrir des choix), donc à améliorer sa qualité de vie.

La deuxième composante vise à empêcher l'escalade des CP (dès les premières manifestations) vers des manifestations plus graves pouvant mener à une crise comportementale et est appelée prévention active. La planification de cette composante comporte l'identification de quelques étapes (souvent de 2 à 3 ) de quatre à cinq étapes d'escalade appréhendée, généralement indiquées par des indices précurseurs observables (chez la personne), et qui doivent être associées à des interventions réactives de désescalade (Tassé, Havercamp et Thompson, 2006). Tout comme pour les aménagements préventifs, on ne vise pas à faire apprendre à la personne de nouveaux comportements. On cherche plutôt à enrayer la progression de la désorganisation comportementale. La prévention active a pour objet d'aider la personne à retrouver son calme ou une certaine disponibilité pour résoudre au mieux la situation problématique. Les moyens d'intervention proposés peuvent consister en de l'écoute active (trouver la cause du stress ou de la frustration, pour la nommer et la gérer) ou en des stratégies de redirection ou de modification des déclencheurs, en passant par de la résolution de problème facilitée (Russell, 2011), c'est-à-dire des suggestions de retour au calme pour trouver des solutions à très court terme, tel qu'offrir des choix (Tassé et al., 2006). 
Et enfin, la dernière composante à mettre en place comporte des interventions d'adaptation/réadaptation et de traitement. Ces actions visent à modifier ou à réduire l'impact des vulnérabilités de la personne. Ce sont des caractéristiques liées à la personne et qui sont associées à une plus grande probabilité d'apparition de TGC chez cette personne. Elles peuvent aussi bien être psychologiques que biologiques et de nature normale ou pathologique (Sabourin et Lapointe, 2014). Par exemple, Iorsqu'une personne présente un seuil de frustration très faible associé à un problème de communication expressif, il serait nécessaire de mettre en place un apprentissage d'habiletés adaptatives ou de remplacement du TGC tel que de demander une pause ou faire des respirations profondes puis demander de l'aide. Relativement à des difficultés de santé physique, mentale ou neurologique, cette composante implique de rechercher un traitement ou une intervention permettant de réduire l'impact fonctionnel de ces problèmes ( $p$. ex., douleur associée). Ces interventions visent donc surtout à augmenter les habiletés adaptatives de la personne et à réduire les impacts négatifs des troubles médicaux ou psychiatriques qui contribuent à l'augmentation de la probabilité de TGC chez celle-ci.

Pour obtenir des résultats optimaux, l'AIMM postule qu'il faut intervenir simultanément sur ces trois différentes composantes, c'est-à-dire sélectionner des actions dans ces trois domaines et les inclure dans un plan d'action global: Plan d'action multimodal en TGC (PAMTGC Formation SQETGC, 2014). Toutefois, la plupart du temps, des aménagements préventifs et la prévention active sont implantés en priorité afin de réduire le stress et les conséquences associées aux manifestations de TGC autant chez la personne que chez l'entourage. Ceci permet de créer de l'espoir sur le potentiel d'amélioration et de mettre en place de meilleures conditions d'apprentissage de nouvelles habiletés, et aussi d'explorer de nouveaux traitements.

Les trois dernières composantes du processus de l'AIMM (les composantes 5 à 7) sont étroitement interreliées par l'entremise de l'articulation et du déploiement du PAMTGC. La composante 5 consiste à organiser le suivi des interventions. Tout dépendant de l'organisation du travail dans l'établissement, les responsabilités des uns et des autres peuvent varier légèrement. Un certain nombre de tâches prédéfinies doivent toutefois être absolument réalisées à cette étape, soit de définir les premières mesures de résultats à documenter (dans une grille de dispersion), former l'entourage de la personne aux principes et aux stratégies d'interventions pertinentes et identifier leurs responsabilités respectives, identifier les professionnels requis pour vérifier les hypothèses nécessitant une évaluation, organiser les rencontres de suivi et les échéanciers associés (voir tâches préalables au suivi des interventions dans Buisson, Rondeau et Sabourin, 2012). La composante 6 représente 
l'application comme telle des interventions planifiées. Enfin, la composante la plus importante et la dernière est le Suivi des interventions. Selon Buisson, Rondeau et Sabourin (2012), ce suivi est un processus systématique de gestion clinique et organisationnelle qui vise à maximiser les effets des interventions pour réduire les manifestations de TGC en s'assurant de l'application conforme du PAMTGC (à l'aide de 5 questions stratégiques), de son ajustement, de la recherche d'autres causes potentielles de TGC, de la concertation de tous les acteurs gravitant autour de la personne, et ceci, tout en s'assurant de l'ajustement des processus cliniques et administratifs aux besoins spécifiques de l'usager. Pour ce faire, ce suivi doit être régulier et soutenu tout en s'appuyant sur la mesure de résultats de l'intervention valides (données objectives permettant de confirmer ou d'infirmer les hypothèses cliniques retenues) (Došen et al., 2007; Gardner et al., 2006).

\section{Efficacité clinique du modèle}

En fait, il y a peu d'études à ce jour qui ont été réalisées sur l'efficacité clinique du modèle AIMM au Québec. Toutefois, à notre connaissance, il y a deux études qui ont été réalisées avec des schèmes quasi expérimentaux et un certain nombre d'études de cas avec des données d'observation en continu qui regroupent au total 66 usagers, d'âge, de diagnostics et de TGC différents.

La première étude a été réalisée par le Dr Jean Caron (2004) qui avait reçu le mandat, comme chercheur indépendant, d'évaluer l'impact clinique du programme expérimental régional d'expertise multidisciplinaire en TGC dans les années 2000. Ce programme utilisait l'AIMM dans le cadre de programmes individualisés d'intervention conjointe intensive ou de consultation ponctuelle. Cette étude s'est déroulée de 2002 à 2003 et a utilisé un schème à séries temporelles avec trois mesures répétées, un taux de base avant l'intervention (T0), 21 mois après la phase d'intervention (T1) et 27 mois après (T2). Diverses mesures furent enregistrées sur le TGC, soit la fréquence, la gravité ainsi que les conséquences et les impacts de TGC. D'autres variables furent mesurées, soit l'état de santé général et mental des personnes ainsi que leur niveau de qualité de vie. Ces personnes étaient de jeunes adultes $(M=23,7$ ans, $\dot{E} T=14,5$ ) et présentaient toutes des TGC, et ce, depuis de nombreuses années en milieu institutionnel $(M=19,3$ ans, $E ́ T=14,9)$. Un total de 29 personnes ont été suivies, mais uniquement 22 ont pu être évaluées aux trois moments de mesure (T0, T1 et T2).

Les résultats de cette étude ont révélé que la gravité de toutes les catégories de TGC, autant pour les TGC internalisés qu'externalisés mesurés à l'Adaptative Behavior Scale-Residential and Community: 2: ABS_RC 2 (Nihira, Leland et Lambert, 1993), a été réduite de façon 
hautement significative sur le plan statistique, particulièrement au niveau des TC externalisés $(F(4,50)=18,37, p<0,000)$. II en est de même sur le plan clinique, une réduction moyenne de $33 \%$ a été relevée au Temps 1 (T1) par rapport au taux de base (T0). Cette réduction s'est d'ailleurs maintenue en T2 (47\%: Temps 2 vs Temps 0$)$. Sur le plan des indices de la santé mentale tels que mesurés au SF-36 Health Survey (Ware et Gandek, 1994), des améliorations statistiquement significatives ont aussi été observées entre le T0 et les T1 et T2 $(F(6,50)=9,23, p<0,000)$. Au niveau de la qualité de vie, les mesures standardisées utilisées (Quality of Life Instrument Package : Raphael, Brown et Renwick, 1999) n'ont pas pu objectiver d'améliorations statistiquement significatives, et ce, même à travers les diverses dimensions.

Une autre étude a été réalisée en 2004-2005, mais cette fois auprès de 14 enfants $(M=8$ ans, $E ́ T=2,83$ ) en milieu scolaire (Sabourin et Lardon, 2006). Tous les enfants présentaient un trouble du spectre de l'autisme (TSA), mais aussi une DI comme diagnostics principaux ainsi qu'un ou plusieurs TGC. La majorité d'entre eux présentaient d'autres diagnostics tels que des troubles attentionnels avec ou sans hyperactivité ou des troubles sévères du langage. Un élève par classe fut sélectionné dans les 16 classes de l'école afin de favoriser l'amélioration de l'expertise dans l'ensemble de l'école et de mesurer les effets cliniques du modèle AIMM auprès des élèves cibles. Diverses mesures furent prises au niveau des TGC cibles, du temps de travail scolaire et deux mesures globales des TC. Un schème de mesure quasi expérimental Pré-Post a aussi été utilisé. Après seulement 8 semaines d'interventions intensives, des améliorations statistiquement significatives ont pu être relevées au niveau des TGC externalisés à l'ABS-RC 2 ( $\mathrm{t}(14)=2,67, p<0,028)$ et sur le plan clinique, avec une moyenne d'amélioration de $39 \%$ avec une fourchette de $21 \%$ à $66,7 \%$. Cette amélioration s'est maintenue après 32 semaines autant au niveau de la fréquence (32\%) à l'ABS-RC 2 qu'au niveau de la gravité des comportements $(36 \%)$, et ce, même après le retrait de la supervision terrain intensive offerte par l'équipe du PREM-TGC de Montréal.

Enfin, des études de cas sur les effets cliniques de l'intervention multimodale et du suivi des interventions auprès de trois cohortes dans trois établissements québécois ont été menées dans les années 2004 à 2009 regroupant au total 30 usagers (Lapointe, 2011; Lapointe et Sabourin, 2010a, 2010b; Lapointe, Sabourin et Bégin, 2012). Ces cohortes d'études de cas uniques avec des données d'observations en continu ont été suivies pendant des durées variant de 14 mois à 5 ans. En plus des mesures d'observation continue, des évaluations subjectives des changements de la gravité du TGC sur un Likert de 1 à 10 et des mesures des TGC à l'EGCP-II (Sabourin et al., 2016) ont été réalisées dans un schéma de recherche Pré-Post. 
Toutes les mesures utilisées ont révélé une réduction importante de la gravité des TGC ciblés après l'application supervisée d'un PAMTGC global. Le niveau de gravité est notamment réduit en moyenne de 1 écart type à l'EGCP-II pour la catégorie de comportements ayant la cote la plus grave pour chacune des personnes participantes. Les données d'observation en continu dans une grille de dispersion indiquent une réduction progressive de la fréquence des manifestations les plus graves de TGC sur une période moyenne de plus de 3 ans. Entre la première et la dernière mesure pour l'ensemble des cohortes, la réduction moyenne de la fréquence du TGC cible est de l'ordre de $70 \%$.

Globalement, les données actuellement accumulées sur l'impact clinique de l'AIMM sont encourageantes, mais pas nécessairement concluantes. En effet, le petit nombre de participants par étude et les faiblesses méthodologiques des diverses études en limitent grandement la portée en tant que données probantes.

Le soutien comportemental positif multiéléments (SCP-ME)

Dans les pays anglo-saxons, tels que les États-Unis, la GrandeBretagne ou l'Australie, le modèle d'intervention le plus souvent préconisé face en TGC est appelé "Positive Behavioral Support" que nous traduirons en français par Soutien Comportemental Positif (SCP). Pour certains auteurs (Johnston, Foxx, Jacobson, Green et Mulick, 2006), ce serait autour des années 2000 que le SCP-ME fut nettement revendiqué comme une discipline à part entière du modèle d'Analyse appliquée du comportement. II serait apparu en appui au mouvement de gestion non aversive du comportement qui aurait débuté dans les années 1980 à 1990 avec des auteurs tels que Horner ou Carr. Horner et ses collègues mentionnaient déjà que le SCP comportait plusieurs éléments distinctifs tels que :

- l'accent mis sur le changement de style de vie de la personne,

- l'évaluation fonctionnelle,

- les interventions multiples ou multiéléments,

- la manipulation des événements écologiques et contributeurs (setting events),

- l'enseignement de comportements adaptatifs,

- la création d'environnements avec des conséquences efficaces,

- la restriction maximale de l'usage de la punition,

- la nette distinction entre les procédures d'intervention d'urgence (de crise) par rapport aux procédures de programmation positive

- et la validation sociale de l'ensemble des interventions sélectionnées pour un usager avec une attention spéciale pour le respect de la dignité de ces personnes (Horner et al., 1990). 
Plus récemment, LaVigna et Willis (2012) ont dirigé une revue des travaux de recherche sur l'efficacité de ce modèle en TGC. D'ailleurs, leur modèle de SCP-ME est souvent cité comme un modèle particulièrement représentatif (LaVigna, Christian et Willis, 2005; LaVigna et Willis, 2005; McClean et Grey, 2007).

Leur modèle postule qu'il faut atteindre six types de résultats (buts ultimes) de façon simultanée, soit 1) l'amélioration de la qualité de vie de la personne, 2) la validité sociale des interventions, 3) la persistance des effets, 4) la généralisation des effets, 5) la réduction des effets secondaires négatifs et 6 ) la vitesse et l'ampleur des effets des interventions dans le temps et sur la gravité des épisodes de TGC (LaVigna et Willis, 2005). Pour ce faire, leur modèle conceptuel comporte quatre grandes étapes, soit une étape d'évaluation fonctionnelle, suivie de l'élaboration d'un plan global de soutien, d'une étape de médiation ou de formation (incluant le soutien aux agents de changement) et, enfin, la mesure de résultats à des niveaux multiples. Pour bien comprendre leur modèle et pouvoir le comparer à l'AIMM, il faut décrire chacune de ces étapes de façon plus détaillée.

Au niveau de l'évaluation, il s'agit d'une évaluation fonctionnelle globale et exhaustive qui nécessite la cueillette d'informations diverses sous la forme d'observations directes des comportements de la personne, d'interactions avec celle-ci, de la lecture des informations contenues dans son dossier et d'entrevues approfondies auprès de l'entourage (LaVigna et al., 2005). Le contenu de cette évaluation couvre tous les domaines de la vie de la personne qui peut avoir une fonction ou donner un sens aux CP de la personne tels que :

- le motif de référence;

- une description complète de la personne à partir de ses caractéristiques physiques jusqu'à ses déficits particuliers (cognitifs, de langage, etc.) en passant par ses capacités adaptatives spécifiques;

- son histoire familiale, son style de vie actuel, ses principales activités, son histoire médicale ou psychiatrique, les traitements reçus ou les interventions tentées, une analyse motivationnelle;

- et, enfin, une analyse fonctionnelle comportementale du TGC qui comporte, entre autres, une description opérationnelle des TC, un historique des TC, une analyse des antécédents incluant les contributeurs et les déclencheurs associés à des périodes de haute probabilité de TC que de faible probabilité, une analyse des conséquents, autant planifiés que non planifiés, et leur effets, dans le temps et sur la sévérité des TC, une analyse écologique permettant d'identifier les inadéquations entre les besoins de la personne et la réponse de son environnement pour ultimement poser des hypothèses 
sur la fonction du TC, particulièrement en termes de fonction de communication, et en considérant surtout le point de vue de la personne (LaVigna et al., 2005).

Cette évaluation permet de formuler des hypothèses fonctionnelles sur lesquelles les interventions pertinentes pourront être basées (McClean et Grey, 2007).

La deuxième étape est l'élaboration d'un plan d'intervention multiéléments (PIM) comportant quatre domaines distincts, soit la mise en place de stratégies dites écologiques, de programmation positive, de soutien spécifique ou de traitement direct et, enfin, de mesures réactives ou de gestion situationnelle de la gravité des TC.

Les stratégies écologiques incluent un ensemble de mesures visant à améliorer l'adéquation entre les caractéristiques de la personne et son environnement afin de faciliter les changements comportementaux de la personne ( $p$. ex, réduire le bruit dans l'environnement qui suscite de l'agitation et de l'irritabilité chez la personne). Elles peuvent inclure une plus grande possibilité de choix ou d'opportunités pour la personne.

Dans le cas de la programmation positive, les stratégies utilisées dans cette section visent à développer des habiletés chez la personne pour l'aider à s'adapter plus facilement à son environnement. Ainsi, à l'aide de procédures d'enseignement systématique, la personne apprendra de nouvelles habiletés et de nouvelles compétences pour mieux fonctionner dans son environnement, lesquelles favoriseront sa participation sociale. Plusieurs types d'habiletés fonctionnelles sont considérés. II y a tout d'abord les habiletés adaptatives en général, dans les activités de la vie de tous les jours. Celles-ci vont aider la personne à fonctionner dans un plus grand nombre d'activités pertinentes autant sur le plan résidentiel que communautaire. Les habiletés équivalentes à la fonction du TC, par exemple d'apprendre un moyen de communiquer une demande de pause avec un moyen alternatif (p. ex., lever une carte) plutôt que de crier ou s'automutiler. Les habiletés fonctionnelles associées comme d'apprendre à relaxer, sur demande, lors de périodes d'attente trop longues plutôt que de se fâcher. Enfin, une des habiletés les plus importantes est souvent d'apprendre à accepter à tolérer un certain nombre de tâches ou de situations. Comme la fonction des TC est souvent d'éviter de faire des tâches, il pourrait être nécessaire d'apprendre à la personne de tolérer la réalisation de certaines tâches, même de façon minimale, dans le cadre d'un programme de renforcement à la collaboration à cette tâche. Cette procédure devrait réduire considérablement la présence de TC lors de l'exécution de telles tâches devant être réalisées de façon régulière (se brosser les dents, participer à desservir sa place à table, etc.). 
La troisième section du PIM comporte des stratégies de soutien spécifique ou de traitement direct du TGC. On fait ici référence surtout à l'utilisation de programmes de renforcement différentiel de comportements alternatifs ou d'autres comportements, mais il est possible d'inclure dans cette section des stratégies de contrôle des antécédents du TGC ou de contrôle des consignes. Ce type de procédures est d'ailleurs reconnu comme pouvant amener une réduction efficace des TC (Harvey et al., 2009).

La dernière section du PIM doit comprendre des mesures réactives lors de l'apparition de TC, mais comportant des stratégies axées sur la réduction, à court terme, de la gravité des TC sans avoir recours à la punition. On pense ici, entre autres, à des stratégies de réduction de la stimulation, de l'écoute active, de la redirection, de la relaxation facilitée ou de la résolution facilitée (McClean et Grey, 2007; Russell, 2011).

La troisième étape du SCP est la médiation qui vise à assurer l'implantation conforme du PIM pour la personne. Ce processus consiste à mettre en place une série de mesures pour, entre autres, former et soutenir adéquatement les différentes parties prenantes (personnes de l'entourage, des professionnels et gestionnaires) sur l'utilisation effective du PIM et la mesure des résultats. Pour ce faire, il y a production, sous forme graphique, des résultats obtenus par rapport aux buts ultimes visés et distribués à toutes les personnes concernées (sur une base hebdomadaire ou mensuelle). Le cas échéant, de la formation sur mesure ou de la supervision terrain est offerte pour favoriser l'application conforme d'un PIM individuel de SCP et pour soutenir les intervenants dans l'utilisation des stratégies recommandées. Ce processus s'avère essentiel pour favoriser la participation de l'entourage de la personne, car l'intégration des changements de pratiques nécessaires à l'efficacité du SCP peut susciter beaucoup de résistance ou de critiques et nécessite des changements importants aux pratiques habituelles de l'entourage des personnes et dans les systèmes de services (Jones, 2013; LaVigna et Willis, 2012). Les diverses stratégies pertinentes à cette étape ont fait l'objet d'une publication appelée Periodic service review qui s'inspire des travaux sur la gestion organisationnelle comportementale visant l'atteinte de standards de qualité totale dans les services sociaux ou éducatifs (LaVigna, Willis, Shaull, Abedi et Sweiter, 1994).

La quatrième étape consiste à mesurer le niveau d'atteinte des résultats dans chacun des domaines visés par les six buts ultimes. Rappelons qu'il s'agit ici de vérifier s'il y a eu amélioration de la qualité de vie de la personne après l'implantation du PIM du SCP, s'il y a eu réduction de la fréquence et de la gravité des TC, si ces changements sont durables et généralisés ou s'il y a eu des effets indésirables à la suite de 
l'intervention et, finalement, si les objectifs fixés et les méthodes utilisées dans le PIM de la personne ont une bonne validité sociale, c'est-à-dire s'ils sont jugés acceptables par la personne, sa famille et les intervenants concernés (LaVigna et al., 2005).

\section{Efficacité du modèle SCP-ME}

Chez les personnes présentant un $\mathrm{DI}$, il existe actuellement un nombre intéressant d'études démontrant l'efficacité du modèle SCP-ME multiéléments pour réduire la fréquence et la gravité du TGC. LaVigna et Willis (2012) ont réussi à identifier 12 études différentes sur l'efficacité du modèle SCP multiéléments regroupant 423 sujets présentant des TGC tels que des comportements d'agressivité physique jusqu'à des comportements d'automutilation. Afin de démontrer le plus clairement possible l'efficacité du SCP, même en l'absence de mesures aversives à proprement parler, les participants cibles des études devaient présenter de véritables TGC et non pas uniquement des TC de moindre gravité tels que des crises de colère sans agressivité physique ou de la non-collaboration.

Ces études ont été sélectionnées en respectant plusieurs critères permettant de ne retenir que les études représentant bien le modèle SCP et l'utilisation des quatre composantes du SCP-ME. De plus, les études retenues satisfaisaient les critères de type 3 de Kazdin (1981) sur les études de cas unique pour pouvoir tirer des conclusions valides à partir des données recueillies, c'est-à-dire qu'elles devaient 1) contenir des données d'observations sur plusieurs comportements simultanément; 2) réaliser des évaluations en continu autant durant la phase du taux de base que dans celle de l'intervention et des phases de suivi ou de relance; 3) cibler les TC cibles relativement chronicisés de façon telle que sans intervention directe et efficace ils ne puissent être diminués ou améliorés facilement; et 4) regrouper des centaines d'usagers d'âge varié, de diagnostics, de niveau fonctionnel, d'historique personnel et de pays d'origine différents.

LaVigna et Willis (2012) ont aussi recensé 28 études de cas individuelles bien contrôlées et réalisées par six équipes différentes, dont seulement trois réalisées par leur équipe professionnelle. Ils ont présenté deux autres types d'études, soit 2 études de formation-supervision de groupes (cohortes) d'intervenants (directs ou de professionnels) par des chercheurs différents qui réalisaient des études de cas bien contrôlées pour un total de 272 usagers, et 2 cohortes de cas contrôlées avec des groupes de comparaison (schéma quasi expérimental) par des équipes différentes de chercheurs regroupant au total 123 usagers.

Toutes ces études ont démontré une diminution hautement significative de la fréquence et du taux de gravité de TGC des participants, 
sauf quelques exceptions, dans les études avec des cohortes importantes d'usagers. Le taux d'efficacité d'amélioration clinique du TGC pouvant aller jusqu'à $70 \%$ d'amélioration moyenne de la fréquence par rapport au taux de base, et ce, pour $77 \%$ du groupe d'usagers suivis dans l'étude $(\mathrm{N}=138)$ de McClean et ses collaborateurs (2005). Ce résultat fut relevé après une période d'intervention moyenne de 18 mois. De plus, plusieurs études de coûts réalisées en parallèle avec certaines des études citées par LaVigna et Willis (2012) tendent à démontrer de façon convaincante une diminution approximative des coûts de $28 \%$ après une intervention de type SCP pour la clientèle en situation de TGC. La robustesse de la démonstration tient aussi au fait que ces études ont été réalisées dans plusieurs pays différents, dont les États-Unis, l'Australie et l'Irlande, et par plusieurs équipes différentes (LaVigna et Willis, 2012, p. 192).

\section{Comparaison de l'AIMM et du SCP Multiéléments}

Compte tenu de la démonstration de l'efficacité clinique du modèle SCP-ME et de l'accumulation des données probantes dans ce sens, le modèle SCP-ME pourrait être reconnu comme une pratique exemplaire en TGC. Selon Sabourin et ses collaborateurs, une pratique serait exemplaire si elle est fondée sur des études quasi expérimentales ou analytiques par observation de qualité moyenne ou élevée et qu'elle soit recommandée par un consensus d'experts de qualité élevée ou moyenne (Sabourin, Couture, Morin et Proulx, 2015 : niveaux de qualité de la preuve, page 16). II serait donc intéressant de réaliser un certain nombre de comparaisons entre les deux modèles afin d'en déduire des pistes d'amélioration possibles de l'AIMM. Une première méthode de comparaison serait de comparer les deux modèles en utilisant les éléments essentiels du modèle SCP-ME (principes et stratégies de base) qui peuvent être reconnus comme efficaces selon les résultats de la revue narrative de LaVigna et Willis (2012).

Pour ce faire, une analyse des écrits sur les principes de SCP-ME (LaVigna et Willis, 2005) et les stratégies utilisées dans les trois cohortes de formation/supervision documentées (Crates et Spicer, 2012; Grey et McLean, 2007; LaVigna et al., 2005) a permis de déceler onze éléments essentiels répartis en six grands domaines associés aux buts ultimes du SCP-ME (voir la liste de la première colonne du Tableau 1: Analyse comparée de l'AIMM par rapport au SCP-ME).

À partir des divers documents et pratiques habituelles associés à l'AIMM, il était possible d'établir un ensemble de ressemblances et de différences entre ce modèle québécois d'intervention et le SCP-ME (voir les colonnes 2 et 3 du Tableau 1). Pour faciliter ce type de comparaison, un Likert d'évaluation qualitative à six niveaux a été élaboré (voir Tableau 2). Essentiellement, ce Likert intègre deux concepts de base qui 
varient sur un continuum de nombre et de qualité des principes ou des stratégies qui sont compatibles avec le modèle de comparaison cible, le SCP-ME. Pour bien mettre l'accent sur les singularités de ce modèle, la colonne 3 du Tableau 1 apporte des précisions sur les particularités du SCP-ME par rapport à l'AIMM.

Sur le plan des principes de base ou des postulats (élément $n^{\circ} 1$ ), les deux modèles comportent globalement les mêmes principes ou postulats, comme le démontre la présentation des deux modèles. II serait donc possible de considérer les deux modèles parfaitement compatibles (niveau 5 de compatibilité) à ce niveau puisqu'ils reposent essentiellement sur les mêmes principes et valeurs de base. La plus grande différence entre les deux réside dans l'inclusion dans l'AIMM de l'ensemble des causalités fonctionnelles possibles du TGC incluant celles d'ordre psychologique et médical pertinentes et non seulement celles liées à l'environnement.

Sur le plan de l'évaluation fonctionnelle globale (élément $n^{\circ} 2$ ), l'AIMM utiliserait la quasi-totalité des stratégies de base du SCP-ME. À ce niveau, il est donc possible de considérer l'AIMM comme parfaitement compatible avec le SCP-ME (niveau 5 de compatibilité). Toutefois, I'AIMM, au contraire du SCP-ME, met un peu moins l'accent sur les forces de la personne dans la grille multimodale et sur la probabilité différentielle de non-apparition du TGC.

Le troisième élément essentiel de SCP-ME est au niveau de la formulation d'hypothèses fonctionnelles (LaVigna et al., 2005). Les deux modèles formulent explicitement des hypothèses fonctionnelles qui facilitent le choix des interventions à privilégier. Dans ce sens, elles sont parfaitement compatibles sur cet élément (niveau 5 de compatibilité).

Dans le contenu du Plan d'Intervention Multiéléments (PIM), les stratégies écologiques sont le quatrième élément $\left(n^{\circ} 4.1\right)$ du tableau de comparaison. Cet élément inclut des recommandations d'ajustement sur le plan de l'environnement physique, interpersonnel et de programmation (Crates et Spicer, 2012) comme dans l'AIMM. Dans l'AIMM, seule l'appellation change (aménagements préventifs) et peut inclure des actions sur les antécédents pour favoriser l'apparition de comportements alternatifs. II y a une compatibilité parfaite à ce niveau (de niveau 5 dans le Likert). Ainsi, les quatre premiers éléments de comparaison étant parfaitement compatibles, il n'y a pas d'indication claire d'améliorations à l'AIMM qui pourrait être déduite de ces premiers constats. 
Tableau 1

Analyse comparée de l'AIMM par rapport au modèle SCP-ME

\begin{tabular}{|c|c|c|c|}
\hline $\begin{array}{l}\text { Éléments essentiels } \\
\quad \text { du SCP-ME }\end{array}$ & $\begin{array}{l}\text { Ressemblances et particularités de l'AIMM vs } \\
\text { le SCP-ME }\end{array}$ & Particularités du SCP-ME vs l'AIMM & $\begin{array}{l}\text { Compatibilité de } \\
\text { l'AIMM avec } \\
\text { le SCP-ME }\end{array}$ \\
\hline $\begin{array}{l}1 \text { - Principes de } \\
\text { base/postulats }\end{array}$ & $\begin{array}{ll} & \text { Mêmes principes de base } \\
\text { - } & \text { Formulation sous forme de valeurs plutôt que } \\
\text { de résultats attendus }\end{array}$ & $\begin{array}{l}\text { Passe sous silence les aspects } \\
\text { fonctionnels d'ordre psychopathologique } \\
\text { et biologique }\end{array}$ & $\begin{array}{l}\text { Parfaitement } \\
\text { compatible }=5\end{array}$ \\
\hline $\begin{array}{l}2 \text { - Évaluation fonctionnelle } \\
\text { globale }\end{array}$ & $\begin{array}{ll}\text { - } & \text { Mêmes stratégies de base } \\
\text { - } & \text { Met beaucoup d'accent sur les aspects } \\
& \text { fonctionnels psychologiques et médicaux. } \\
\text { - } \quad \text { Met moins l'accent sur les forces }\end{array}$ & $\begin{array}{l}\text { Inclut davantage les forces et la } \\
\text { probabilité différentielle de non- } \\
\text { apparition des TGC } \\
\text { Absence des aspects fonctionnels } \\
\text { biologiques et psychopathologiques }\end{array}$ & $\begin{array}{l}\text { Parfaitement } \\
\text { compatible }=5\end{array}$ \\
\hline $\begin{array}{l}3 \text { - Formulation } \\
\text { d'hypothèses } \\
\text { fonctionnelles }\end{array}$ & $\begin{array}{ll} & \text { Même type d'hypothèses } \\
\text { - } & \text { Modalités de travail plus développées et } \\
& \text { fonctionnelles (grille d'analyse MM) }\end{array}$ & $\begin{array}{l}\text { Formulations fonctionnelles explicites en } \\
\text { lien avec les TGC }\end{array}$ & $\begin{array}{l}\text { Parfaitement } \\
\text { compatible }=5\end{array}$ \\
\hline $\begin{array}{l}4 \text { - Plan d'intervention } \\
\text { 4.1 Stratégies } \\
\text { écologiques }\end{array}$ & $\begin{array}{l}\text { - Présence des mêmes stratégies, mais } \\
\text { nommées plutôt des aménagements } \\
\text { préventifs } \\
\text { - Contenu plus large et inclut souvent le } \\
\text { contrôle des antécédents et des consignes }\end{array}$ & $\begin{array}{l}\text { Exclut le contrôle des antécédents et } \\
\text { des consignes qui se retrouve dans le } \\
\text { traitement direct }\end{array}$ & $\begin{array}{l}\text { Parfaitement } \\
\text { compatible }=5\end{array}$ \\
\hline $\begin{array}{l}\text { 4.2 Programmation } \\
\text { positive }\end{array}$ & $\begin{array}{l}\text { - Présence d'une majorité des mêmes } \\
\text { stratégies } \\
\text { - Formulation plus large et moins explicite (plus } \\
\text { floue) des procédures suggérées }\end{array}$ & $\begin{array}{l}\text { Procédures plus explicites et plus } \\
\text { nombreuses avec des procédures } \\
\text { spécifiques d'apprentissage }\end{array}$ & $\begin{array}{l}\text { Moyennement } \\
\text { compatible }=3\end{array}$ \\
\hline $\begin{array}{l}\text { 4.3 Traitement direct } \\
\text { des TGC }\end{array}$ & $\begin{array}{l}\text { - Présence d'une majorité des mêmes } \\
\text { stratégies } \\
\text { - Insiste sur les autres causes et moins sur les } \\
\text { comportements alternatifs } \\
\text { - Moins explicite (plus floue) sur les processus } \\
\text { d'apprentissage }\end{array}$ & $\begin{array}{l}\text { Procédures plus explicites avec des } \\
\text { procédures spécifiques d'apprentissage }\end{array}$ & $\begin{array}{l}\text { Moyennement } \\
\text { compatible }=3\end{array}$ \\
\hline
\end{tabular}


Tableau 1

Analyse comparée de l'AIMM par rapport au modèle SCP-ME (suite)

\begin{tabular}{|c|c|c|c|}
\hline $\begin{array}{l}\text { Éléments essentiels } \\
\text { du SCP-ME }\end{array}$ & $\begin{array}{l}\text { Ressemblances et particularités de l'AIMM vs } \\
\text { le SCP-ME }\end{array}$ & Particularités du SCP-ME vs l'AIMM & $\begin{array}{l}\text { Compatibilité de } \\
\text { l'AIMM avec } \\
\text { le SCP-ME }\end{array}$ \\
\hline $\begin{array}{l}\text { 4.4 Stratégies } \\
\text { réactives de } \\
\text { désescalade }\end{array}$ & $\begin{array}{l}\text { - Présence d'une très grande partie des } \\
\text { stratégies } \\
\text { - Actions mises en séquence hiérarchique }\end{array}$ & $\begin{array}{l}\text { Stratégies retenues peu nombreuses } \\
\text { Moins mises en séquence hiérarchique }\end{array}$ & $\begin{array}{l}\text { Globalement } \\
\text { compatible }=4\end{array}$ \\
\hline $\begin{array}{l}5 \text { - Médiation } \\
5.1 \text { Formation sur le } \\
\text { modèle }\end{array}$ & $\begin{array}{l}\text { - Présence généralement d'une formation de } \\
\text { base et d'un peu de supervision } \\
\text { - D'une durée de } 3 \text { à } 5 \text { jours } \\
\text { - Le coaching est généralement faible }\end{array}$ & $\begin{array}{l}\text { Généralement d'une durée de } 9 \text { à } 13 \\
\text { jours, ce qui inclut les procédures } \\
\text { d'enseignement et de plusieurs sessions } \\
\text { de coaching }\end{array}$ & $\begin{array}{l}\text { Globalement } \\
\text { compatible }=4\end{array}$ \\
\hline $\begin{array}{l}\text { 5.2 Supervision sur } \\
\text { une situation réelle }\end{array}$ & $\begin{array}{l}\text { - Présence d'une majorité des mêmes } \\
\text { stratégies } \\
\text { - Uniquement lors de la formation de base } \\
\text { - Manque de coaching individuel, la plupart du } \\
\text { temps }\end{array}$ & $\begin{array}{l}\text { Plusieurs moments de supervision en } \\
\text { individuel et en groupe sont } \\
\text { généralement fournis pendant plusieurs } \\
\text { mois (6) après la formation de base }\end{array}$ & $\begin{array}{l}\text { Moyennement } \\
\text { compatible }=3\end{array}$ \\
\hline $\begin{array}{l}\text { 5.3 Mesure de la } \\
\text { conformité des } \\
\text { interventions }\end{array}$ & $\begin{array}{l}\text { - Présence d'une majorité des mêmes } \\
\text { stratégies } \\
\text { - Questionnement verbal lors des rencontres de } \\
\text { suivi des interventions ( } 5 \text { questions de } \\
\text { conformité) }\end{array}$ & $\begin{array}{l}\text { Mesures de conformité et rétroaction } \\
\text { sous forme de graphiques }\end{array}$ & $\begin{array}{l}\text { Moyennement } \\
\text { compatible }=3\end{array}$ \\
\hline $\begin{array}{l}6 \text { - Mesure des résultats en } \\
\text { continu }\end{array}$ & $\begin{array}{l}\text { - Présence des mêmes stratégies } \\
\text { - Se fait avec un système d'enregistrement } \\
\text { informatisé de données (grille de dispersion) }\end{array}$ & $\begin{array}{l}\text { Mesures d'observation en continu de la } \\
\text { gravité des incidents de TGC }\end{array}$ & $\begin{array}{l}\text { Parfaitement } \\
\text { compatible }=5\end{array}$ \\
\hline
\end{tabular}


Tableau 2

Échelle d'évaluation qualitative de compatibilité de l'AIMM avec le SCP-ME

\begin{tabular}{ll}
\hline Il y a dans l'AIMM : \\
\hline $0 \quad \begin{array}{l}\text { une absence complète des principes et des stratégies constitutives du SCP-ME : le } \\
\text { modèle est nettement incompatible }\end{array}$ \\
$1 \quad \begin{array}{l}\text { un certain nombre des principes ou des stratégies sont plutôt incompatibles avec les } \\
\text { éléments constitutifs du SCP-ME }\end{array}$ \\
$2 \quad \begin{array}{l}\text { un faible nombre des principes ou des stratégies constitutives du SCP-ME : le } \\
\text { modèle est légèrement compatible }\end{array}$ \\
$\begin{array}{l}\text { un bon nombre (une majorité) de principes ou de stratégies constitutives du SCP- } \\
\text { ME : le modèle est moyennement compatible }\end{array}$ \\
$\begin{array}{l}\text { une très grande partie des mêmes principes ou des stratégies constitutives du SCP- } \\
\text { ME : le modèle est globalement compatible }\end{array}$ \\
$5 \quad \begin{array}{l}\text { les mêmes principes ou stratégies constitutives du SCP-ME : le modèle est } \\
\text { parfaitement compatible }\end{array}$ \\
\hline
\end{tabular}

Les cinquième (4.2) et sixième (4.3) éléments sont la programmation positive et le traitement direct du TGC du PIM. La programmation active du SCP-ME comporte quatre types d'habiletés spécifiques à développer, entre autres, des habiletés fonctionnelles équivalentes de remplacement au TC et des habiletés de tolérance ou d'adaptation à son environnement naturel. Dans l'AIMM, la formation insiste elle aussi sur toutes les habiletés déficitaires en général, mais en étant beaucoup moins spécifique sur le type d'habiletés à développer, en particulier en ce qui a trait aux habiletés fonctionnelles équivalentes au TGC. II serait possible de considérer que sur cet aspect, l'AIMM est moyennement compatible avec les recommandations du SCP-ME, donc qualitativement de niveau 3 dans le Likert. II y aurait donc lieu d'inclure dans l'AIMM plus de spécificités dans les choix des habiletés de remplacement qui sont d'ailleurs reconnues comme plus efficaces, car plus reliées aux fonctions de TC visé (Harvey et al., 2009).

Quant au traitement direct du TGC (élément $n^{\circ} 4.3$ ), il y a quatre stratégies qui sont mentionnées, soit le contrôle des stimuli, le contrôle des antécédents, le contrôle des instructions et les programmes de renforcement différentiel de divers types. Dans l'AIMM, il n'y a que les stratégies de renforcement différentiel qui ne sont pas indiquées de façon explicite, les quatre autres sont incluses dans les aménagements préventifs, donc la très grande majorité des stratégies s'y retrouve, mais la plus importante y est absente. En effet, cette stratégie est la seule qui se propose d'enseigner activement à la personne un comportement véritable 
mieux adapté comme alternative au TGC, et ce, dans une perspective d'autocontrôle. II y aurait lieu de considérer cet élément comme moyennement compatible (un score de 3). L'AIMM aurait intérêt à inclure explicitement cet élément dans son processus clinique.

La présence de stratégies réactives de désescalade (élément $n^{\circ} 4.4$ ) est le septième élément de comparaison. Celles-ci sont en très bonne partie présentes dans la formation AIMM, sauf qu'elles sont utilisées de façon hiérarchique, ce qui n'est pas nécessairement le cas dans le SCP$M E$. Le niveau de description des meilleures stratégies à utiliser dans le SCP-ME n'est pas suffisamment précis pour en déduire une recommandation d'amélioration de l'AIMM. Obtenant un score de compatibilité de 4 , il est donc possible de considérer l'AIMM comme globalement compatible.

Sur le plan des variables intermédiaires de l'intervention (Mediation: LaVigna et al., 2005), cette section comprend trois éléments essentiels : la formation d'un intervenant principal sur le modèle (5.1), la supervision individuelle concernant une situation réelle de TGC (5.2) et la mesure de l'utilisation conforme des interventions (5.3) sélectionnées. Les deux modèles préconisent de mettre bien en place un processus formel de formation pour l'entourage (intervenants responsables) afin de faciliter l'implantation de toutes les composantes de leur modèle. Toutefois, dans le cas du SCP-ME, ce processus de formation est d'une durée variant entre 9 à 13 jours de formation et inclus l'enseignement sur les processus d'apprentissage des personnes. La durée de la formation de l'AIMM comporte quant à elle de 3 à 5 jours de formation et insiste surtout sur les principes et stratégies à privilégier avec diverses situations réelles de TGC. Sur le plan de la formation, l'AIMM peut être considérée comme étant moyennement compatible avec le SCP-ME et se voir attribuer un score de 3 comme évaluation qualitative de compatibilité. Le modèle AIMM gagnerait à s'inspirer du modèle du SCP-ME sur cet aspect en augmentant le nombre de journées de formation et à réviser son contenu pour inclure davantage d'informations sur les stratégies d'enseignement pertinentes.

Quant à la supervision professionnelle sur une situation réelle de TGC (élément 5.2), les deux modèles comportent le choix de situations réelles de TGC lors de la formation formelle. Toutefois, le SCP-ME va plus loin en intégrant, pour chacun des participants, une situation réelle d'une personne dont ils sont directement responsables. De plus, cette situation fait l'objet d'un coaching individuel ou de groupe à plusieurs reprises après la formation formelle. Dans le cas de l'AIMM, ce type de coaching peut être offert, mais cette étape n'est pas toujours présente. Sur le plan de la compatibilité, l'AIMM pourrait être considérée comme moyennement compatible avec le SCP-ME (un score de 3) étant donné qu'une majorité 
des stratégies pertinentes sont présentes, mais qu'elles le sont dans des proportions différentes. Le coaching individualisé dans une situation réelle de TGC de chacun des participants à la formation AIMM serait une bonne piste d'amélioration à inclure dans le processus clinique du modèle multimodal.

Concernant la mesure de la conformité des interventions, le SCP-ME s'assure de quantifier l'application conforme des interventions planifiées et de fournir à l'entourage d'une personne une rétroaction dans ce sens sous forme graphique. L'AIMM fait un suivi à ce niveau, mais sous forme davantage verbale lors de réunions avec l'entourage. Ce modèle d'intervention privilégie l'utilisation systématique de cinq questions aux intervenants sur la qualité et la conformité des interventions prévues au plan d'action multimodal (PAMTGC). Sur le plan qualitatif, ces modalités différentes ont littéralement la même fonction sans toutefois être équivalentes. Par contre, elles pourraient être considérées comme moyennement compatibles (score de 3 ) sur le plan fonctionnel. La quantification de la conformité est une piste intéressante d'amélioration, car la seule rétroaction à l'entourage peut stimuler énormément la motivation de celui-ci à atteindre une meilleure conformité des interventions privilégiées.

Quant à la mesure des résultats en continu (élément $\mathrm{n}^{\circ} 6$ ), les deux modèles comportent des modalités passablement équivalentes à ce niveau. L'AIMM utilise une compilation des résultats d'observations quotidiennes à l'intérieur d'une grille de dispersion. Le SCP-ME propose une série de mesures d'observation en continu sur la gravité des incidents de TGC. II est possible de considérer ces deux méthodes comme parfaitement compatibles, donc équivalant à un score de 5 de compatibilité.

Globalement, la présente analyse comparée des deux modèles (I'AIMM par rapport au SCP-ME) permettrait d'établir un pourcentage de compatibilité entre les deux modèles de façon qualitative. Pour ce faire, il devient possible de calculer un score total des scores de compatibilité des 11 éléments de comparaison retenus et de diviser ce score par le total des scores possibles des onze éléments présents dans le tableau 1. Ce résultat multiplié par 100 donnera le pourcentage de compatibilité qualitative globale de l'AIMM par rapport au SCP-ME. Le calcul suivant peut être établi : le score total des 11 éléments de comparaison étant de 44 sur un score total possible de 55, multiplié par 100 égal $80 \%$ $([44 / 55] \times 100=80 \%)$. Ainsi, il est possible de conclure qu'approximativement $80 \%$ des éléments importants qui caractérisent le modèle SCP-ME de LaVigna et ses collaborateurs (2005) sont compatibles avec l'AIMM préconisée au Québec par rapport aux TGC chez les 
personnes présentant une DI. Compte tenu de la qualité des données probantes associées au SCP-ME, il a été possible de déduire des $20 \%$ des scores de compatibilité manquants des pistes intéressantes d'amélioration de l'AIMM. Ces améliorations, une fois intégrées à la pratique habituelle de ce modèle d'intervention, pourraient sans doute générer une plus grande efficacité clinique en termes de réduction de TGC et de leurs impacts.

\section{CONCLUSION}

La présente analyse comparée a permis de mettre en évidence un niveau élevé de qualité du SCP-ME en termes de données probantes par rapport à l'AIMM. Ce dernier modèle d'intervention en TGC a fait l'objet de peu d'études. Les quelques études réalisées au Québec ont généré des résultats intéressants sur le plan clinique quoiqu'elles comportaient un nombre limité de participants et de faiblesses, entre autres sur le plan de la validité interne. Une des faiblesses les plus importantes était de ne pas comporter une description détaillée du processus clinique appliqué et des éléments typiques d'interventions réalisées. En comparant le processus de formation de l'AIMM aux principaux éléments caractéristiques du SCP-ME décrits dans les diverses cohortes de supervision étudiées, il fut possible de discerner un certain nombre de compatibilités entre ces deux modèles, mais surtout de déduire un certain nombre d'améliorations possibles au niveau de l'AIMM.

À la suite de ces constats, les principales recommandations d'améliorations sont concentrées sur le plan d'action multimodal qui devrait être plus spécifique sur les éléments de programmation positive et de traitement direct du TGC et sur les variables intermédiaires. Les enseignements d'équivalents fonctionnels incompatibles avec le TGC et les habiletés connexes (p. ex., relaxation) devraient être davantage priorisés ainsi que les stratégies de renforcement différentiel pour diminuer la probabilité relative du TGC chez les personnes. Au niveau des variables intermédiaires, les recommandations sont d'augmenter la durée de la formation, surtout dans le domaine de la supervision individualisée avec une situation réelle de TGC dans laquelle un intervenant principal est directement concerné. Le contenu devrait aussi porter davantage sur les habiletés d'enseignement pertinentes à la programmation positive et sur le traitement direct du TGC. Enfin, la dernière recommandation concerne l'utilisation de mesures davantage quantitatives pour informer l'entourage sur la conformité de l'utilisation des stratégies inscrites au plan d'intervention de la personne.

La présente analyse contient d'importantes limitations. D'une part, elle ne comporte qu'une seule direction de comparaison, c'est-à-dire que 
l'AIMM n'est comparée qu'aux caractéristiques principales du modèle du SCP-ME. Cette façon de faire peut laisser dans l'ombre plusieurs aspects importants de l'AIMM, surtout que les sources d'information pour les deux modèles sont différentes quoiqu'elles proviennent d'une documentation formelle de formation, dans le cas de l'AIMM, et de processus d'intervention systématique de recherche dans le cas du SCP-ME. De plus, la présente analyse repose sur des critères qualitatifs et plutôt subjectifs étant donné qu'elle inclut seulement le point de vue des auteurs. II aurait été préférable de soumettre cette analyse qualitative à un nombre plus grand de spécialistes afin de pouvoir établir un consensus plus valide. Cette première comparaison vise donc à susciter davantage la réflexion et à stimuler des travaux dans ce sens.

Sur le plan de la recherche, il ne semble pas y avoir de consensus solide, pour l'instant, sur la méthodologie d'évaluation de la qualité de la preuve scientifique et clinique (données probantes) de l'efficacité des traitements en DI (Campbell, Robertson et Jahoda, 2014; Kaiser et Mclntyre, 2010). Ceci peut s'expliquer en partie par l'hétérogénéité de la clientèle DI, car les études réalisées comportent presque exclusivement des études de cas individuelles avec plus ou moins de contrôle des biais possibles (Kaiser et Mclntyre, 2010). Mais la question la plus importante selon Campbell et ses collègues (2014) devrait être de savoir quelles sont les interventions qui fonctionnent le mieux dans la pratique courante de tous les jours et pour qui! Dans ce sens, les questions de validité externe et la puissance de l'effet (clinique) seraient donc les plus importantes, sans toutefois négliger la validité interne d'une recherche sur l'efficacité d'un modèle clinique en TGC. Par exemple, dans les recherches sur le SCP$\mathrm{ME}$, le modèle inclut systématiquement des mesures d'application conforme des stratégies identifiées au plan d'intervention. Ainsi, certaines des recherches citées par LaVigna et Willis (2012) excluent les résultats des participants pour qui le taux d'application conforme est trop faible comme dans l'étude de McClean et ses collaborateurs (2005). Leur pourcentage global d'efficacité clinique peut, sans aucun doute, en être augmenté de façon importante par rapport à d'autres études qui ne contrôlent pas ce facteur, comme dans les quelques études réalisées sur I'AIMM. II faut donc tenir compte de ce type de critère pour évaluer les différentes études.

Concernant le modèle d'AIMM, la priorité de recherche serait donc de réaliser des études de cas multiples dans le cadre de cohortes de supervision d'intervenant principal de personnes présentant une DI et étant en situation de TGC. Ce type d'étude pourrait permettre de mesurer plus adéquatement sur le plan scientifique l'efficacité de l'AIMM et de bien décrire son processus clinique typique ainsi que les stratégies qu'elle priorise. Pour ce faire, une telle étude pourrait s'inspirer des travaux déjà 
réalisés avec des cohortes de supervision sur le modèle SCP-ME dans divers pays.

\section{RÉFÉRENCES}

Allen, D., Lagthorne, P., Tonge, B., Emerson, E., McGill, P., Fletcher, R.,... Kennedy, C. (2013). Towards the prevention of behavioural and psychiatric disorders in people with intellectual disabilities. Journal of Applied Research in Intellectual Disabilities, 26(6), 501-514.

Buisson, D., Rondeau, M. et Sabourin, G. (2012). Le suivi des interventions en troubles graves du comportement (TGC) pour les personnes ayant une DI ou un TED: Guide technique. Montréal, Québec : SQETGC/FQCRDITED.

Campbell, M., Robertson, A. et Jahoda, A. (2014). Psychological therapies for people with intellectual disabilities: comment on a Matrix of evidence for interventions in challenging behaviour. Journal of Intellectual Disabilities Research, 58(2), 172-188.

Caron, J. (2004). L'évaluation de l'impact sur la clientèle du programme régional d'expertise multidisciplinaire en troubles graves du comportement. Rapport présenté à l'Agence de développement de réseaux locaux de services de santé et de services sociaux de Montréal.

Coffre à outils de l'éducateur en TC/TGC (2015). Repéré à http:///www.sqetgc.org/coffre

Crates, N. et Spicer, M. (2012). Developing behavioural training services to meet defined standards within an Australian statewide disability services system and the associated client outcomes. Journal of Intellectual \& Developmental disability, 37(3), 196-208.

Došen, A., Gardner, W. I., Griffiths, D. M., King, R. et Lapointe, A. (2007). Practice guidelines and principles: Assessment, diagnosis, treatment, and related support services for persons with intellectual disabilities and problem behaviour. Tielweg, Pays-Bas : Centre of Consultation and Expertise.

Formation AIMM-SQETGC (2014). Analyse et intervention multimodales en trouble graves du comportement. Partie 1: Principes d'intervention et grille d'analyse multimodale contextuelle. Montréal, Québec: SQETGC/Centre intégré universitaire de santé et de services sociaux de la Mauricie-et-du-Centre-du-Québec.

FQCRDITED (2010). Les services d'adaptation et de réadaptation auprès des personnes ayant un trouble grave du comportement (TGC) : Guide de pratique. Montréal, Québec : Fédération québécoise des CRDITED.

Fraser, D. et Labbé, L. (1993). L'approche positive de la personne... Une conception globale de l'intervention. Laval, Québec : Édition Agence d'Arc et les Éditions de la collectivité.

Gardner, W. I. (2002). Agression and other disruptive behavioral challenges: biomedical and psychosocial assessment and treatment. Kingston, NY : NADD Press.

Gardner, W. I., Došen, A., Griffiths, D., King, R. et Lapointe, A. (2006). Practice guidelines for diagnostic, treatment and related support services for persons with developmental disabilities and serious behavioral problems. Kingston, NY : NADD Press.

Gardner, W. I., Griffiths, D. M. et Hamelin, J. P. (2012). Biopsychosocial features influencing aggression: A multimodal assessment and therapy approach. Dans J. K. Luiselli (dir.), The handbook of high-risk challenging behaviors in people with intellectual and developmental disabilities (p. 83-102). Baltimore, MD : Paul H. Brookes Publishing Co.

Gimpel Peacock, G. et Collett, B.R. (2010). Collaborative home/school interventions: Evidence-based solutions for emotional, behavioral, and academic problems. New York, NY : The Guilford Press.

Grey, I. M. et McClean, B. (2007). Services user outcomes of staff training in positive behaviour support using person-focused training: A control group study. Journal of Applied Research intellectual Disabilities, 20(1), 6-15.

Griffiths, D. M., Gardner, W. I. et Nugent, J. A. (1998). Behavioral supports: individual centered interventions-A multimodal functional approach. Kingston, NY : NADD Press.

Guimond, R., Paquet, M. et Bigras, M. (2016). La communauté de pratique provinciale en troubles graves du comportement: un dispositive d'innovation qui a su s'adapter à la 
transformation de réseau. Le point en santé et services sociaux, 12(4), 54-59. Récupéré de http://sqetgc.org/wp-content/uploads/2017/02/Article-12.04.10-Guimond-coll..pdf

Harvey, S. T., Boer, D., Meyer, L. H. et Evans, I. M. (2009). Updating a meta-analysis of intervention research with challenging behaviour: Treatment validity and standards of practice. Journal of Intellectual and Developmental Disability, 34(1), 67-80.

Horner, R. H., Dunlap, G., Koegel, R. L., Carr, E. G., Sailor, W., Anderson, J.,... O'Neill, R. E. (1990). Toward a technology of "nonaversive » behavioral support. Research and practice for Persons with Severe Disabilities, 15, 125-132.

Johnston, J. M., Foxx, R. M., Jacobson, J. W., Green, G. et Mulick, J. A. (2006). Positive behavior support and applied behavior analysis. The Behavior Analyst, 29(1), 51-74.

Jones, E. (2013). Back to the future: developing competent residential services for people with intellectual disabilities and challenging behaviour. Advances in Mental Health and Intellectual Disabilities, 7(1), 5-17.

Kaiser, A. P. et McIntyre, L. L. (2010). Introduction to special section on evidence-based practices for persons with intellectual and developmental disabilities. American Journal on Intellectual and Developmental Disabilities, 115(5), 357-363.

Kazdin, A. E. (1981). Drawing valid inferences from case studies. Journal of Consulting and Clinical Psychology, 49(2), 183-192.

Lapointe, A. (2011). Évaluation du suivi des interventions en troubles graves du comportement. Présentation au Colloque du SQETGC.

Lapointe, A. et Sabourin, G. (2010a). Monitoring Interventions on Problem Behavior: 5 Years of Experience. The NADD $27^{\text {th }}$ Annual Conference and Exhibit show.

Lapointe, A. et Sabourin, G. (2010b). Monitoring des interventions en troubles graves du comportement. Présenté au Xle congrès international de l'AIRHM, Mons, Belgique.

Lapointe, A., Sabourin, G. et Bégin, D. (2012). Le projet de développement de l'expertise. Un processus efficace de changement des pratiques dans lintervention sur les TGC. AIRHM Congrès 2012.

LaVigna, G. W., Christian, L. et Willis, T. J. (2005). Developing behavioural services to meet defined standards within a national system of specialist education services. Pediatric Rehabilitation, 8(2), 144-155.

LaVigna, G. W. et Willis, T. J. (2005). A positive behavioural support model for breaking the barriers to social and community inclusion. Learning Disability Review, 10(2), 16-23.

LaVigna, G. W. et Willis, T. J. (2012). The efficacy of positive behavioural support with the most challenging behaviour: The evidence and its implications. Journal of Intellectual \& Developmental Disability, 37(3), 185-195.

LaVigna, G. W., Willis, T. J., Shaull, J. F. Abedi, M. et Sweitzer, M. (1994). The periodic service review: A total quality assurance system for human services and education. Baltimore, MD : Paul Brookes Publishing. Co.

McClean, B., Dench, C., Grey, I., Shanahan, S., Fitzsimons, E., Hendler, J. et Corrigan, M. (2005). Person focused training: A model for delivering positing behavioural supports to people with challenging behaviours. Journal of Intellectual Disability Research, 49(5), 340-352.

McClean, B. et Grey, I. (2007). Modifying challenging behaviour and planning positive supports. Dans A. Carr, G. O'Reilly, P. N. Walsh et J. McEvoy (dir.), The handbook of intellectual disability and clinical psychology practice (p. 643-684). New York, NY: Routledge Taylor and Francis group.

Morin, I., Sabourin, G., Labbé, L. et Beaulieu, Y. (2015). Inventaire des causes probables des troubles graves du comportement: manuel de l'utilisateur. Montréal, Québec: SQETGC/Centre intégré universitaire de santé et de services sociaux de la Mauricie-etdu-Centre-du-Québec.

Nihira, K., Leland, H. et Lambert, N. (1993). Adaptative behavior scale-residential and community ( $2^{\mathrm{e}}$ éd.). Austin, TX: Pro-ED- An international Publisher.

Raphael, D., Brown, I. et Renwick, R. (1999). Psychometric properties of the full and short versions of the Quality of Life Instrument Package: Results from the Ontario provincewide study. International Journal of Disability, Development and Education, 46(2), 157168. 
Roberge, M. (2015). Le modèle de plan de développement de l'expertise en TGC. Tome I-Guide pour l'amélioration progressive du dispositif de services en TGC dans les établissements desservant une clientèle ayant une DI ou un TSA et un TGC. Montréal, Québec : SQETGC | CIUSSS de la Mauricie-et-du-Centre-du-Québec.

Russell, L. (2011). Reactive management strategies. Dans S. Hardy et J. Joyce (dir.), Challenging behaviour and people with learning disabilities: $A$ handbook. (p. 65-72). Brighton, Grande-Bretagne : Pavillon Publishing.

Sabourin, G., Couture, G., Morin, D. et Proulx, R. (2015). Intervention de crise auprès de personnes ayant une déficience intellectuelle avec ou sans troubles envahissants $d u$ développement: une recension critique des écrits en consultation avec les parties prenantes. Les collections de l'Institut universitaire en DI et en TSA. Centre intégré de santé et de services sociaux de la Mauricie-et-du-Centre-du-Québec.

Sabourin, G. et Lapointe, A. (2014). Analyse et intervention multimodales en troubles graves du comportement: Grille et lexique. Montréal, Québec : SQETGC | FQCRDITED.

Sabourin, G. et Lardon, Y. (2006). Intervention en milieu scolaire sur les TGC d'élèves ayant un TED : une approche multimodale et systémique. AAMR-Sommet 2006 Montréal.

Sabourin, G., Senécal, P. et Paquet, M. (2016). EGCP-II-R - Échelle d'évaluation des comportements problématiques II, révisée (Manuel de l'utilisateur). Montréal, Canada. SQETGC | CIUSSS de la Mauricie-et-du-Centre-du-Québec.

SQETGC (2017). Protocole d'identification des personnes présentant une DI ou un TSA et manifestant un TGC. Cadres de référence et Guides techniques. Montréal, Québec: SQETGC Centre intégré universitaire de santé et de services sociaux de la Mauricie-etdu-Centre-du-Québec.

Tassé, M. J., Havercamp, S. M. et Thompson, C. (2006). Practice guidelines in working with individuals who have developmental disabilities. Concord, $\mathrm{NH}$ : $\mathrm{PBH}$.

Tassé, M. J., Sabourin, G., Garcin, N. et Lecavalier, L. (2010). Définition d'un trouble grave du comportement chez les personnes ayant une déficience intellectuelle. Canadian Journal of Behavioural Science, 42(1), 62-69.

Ware, J. E. Jr. et Gandek, B. (1994). The SF-36 Health Survey: development and use in mental health research and the IQOLA project. International Journal of Mental Health, 23, 49-73.

\section{RÉSUMÉ}

Le présent article vise à comparer deux modèles d'intervention en TGC, soit le modèle québécois d'Analyse et d'Intervention MultiModales (AIMM) et le modèle de Soutien Comportemental Positif (SCP : Positive Behavioral Support) chez les personnes présentant une déficience intellectuelle (DI). Ce modèle de LaVigna et Willis (2012) a d'ailleurs fait l'objet récemment de démonstrations convaincantes sur le plan de son efficacité et de son efficience clinique. Après avoir constaté les compatibilités et les différences entre ces deux modèles sur le plan qualitatif, certaines pistes d'amélioration clinique et de recherche concernant l'AIMM sont relevées.

\section{MOTS CLÉS}

déficience intellectuelle, trouble du comportement, analyse et intervention.

\section{ABSTRACT}

The purpose of this article is to compare two models of intervention in SBD: the Quebec Multimodal Analysis and Intervention Model (AIMM) and the Positive Behavioral Support Model (SCP) in people with intellectual disabilities (ID). The latter model of LaVigna and Willis (2012) has recently been the subject of convincing demonstrations in terms of its effectiveness and clinical efficiency. After having noted the compatibilities and the differences 
Modèles d'intervention en TGC

between these two models in terms of quality, some lines of clinical improvement and research concerning the AIMM are raised.

\section{KEY WORDS}

intellectual disability, behavioral disorder, analysis and intervention. 